\title{
Molekulardiagnostische Tests und zielgerichtete Therapien
}

Durch die Entwicklung der personalisierten Medizin können in der Therapie der Krebserkrankungen deutliche Fortschritte erreicht werden.

Verschiedene Patienten mit der gleichen klinischen Diagnose können unterschiedlich auf ein und dieselbe Therapie ansprechen, da krankheitsbezogene und krankheitsunabhängige individuelle Merkmale der Patienten die Wirksamkeit der Medikamente beeinflussen können. Daher versucht Roche, Diagnostika und Therapeutika zu entwickeln und bereitzustellen, die eine personalisierte Medizin ermöglichen können - mit dem Ziel, eine Verbesserung der Gesundheit, Lebensqualität und Überlebensdauer der Patienten zu erreichen. Das Konzept der personalisierten Medizin basiert auf neuen molekularen Erkenntnissen zur Krankheitsentstehung und verwendet dabei sowohl molekulardiagnostische Tests als auch zielgerichtete Substanzen, die eine genauere Abstimmung der Therapie und eine bessere Kontrolle der Krankheit ermöglichen können.

\section{Metastasierende Melanome}

Ein Beispiel für die personalisierte Behandlung bei Patienten mit metastasierenden Melanomen stellte Gabriele Beer, Roche Penzberg, vor.

Bei nahezu 50\% der Patienten mit metastasierenden Melanomen können Mutationen des BRAF-Gens nachgewiesen werden. Ein neuer diagnostischer Test identifiziert BRAF-Mutationen, den Angriffspunkt des selektiven RAF-Mutationsinhibitors RG7204 (Abb.). Laut Beer zeigen Ergebnisse früher Studien mit RG7204 (data on

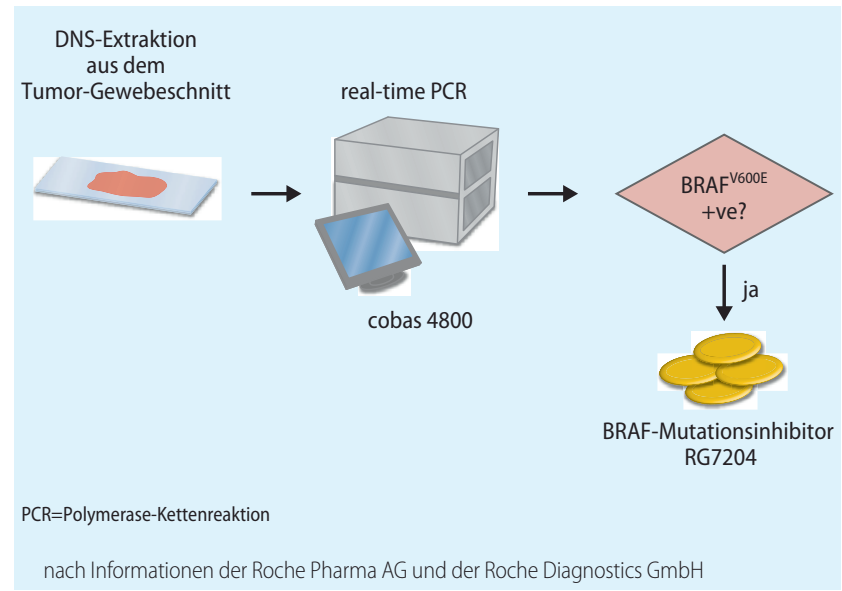

Identifikation der BRAF-Mutation durch einen diagnostischen Test

file) eine Ansprechquote von 81\%, ein progressionsfreies Überleben von > 6 Monaten und ein häufig schnelles Ansprechen.

In einer Phase-III-Studie wird RG7204 derzeit bei Patienten mit metastasierenden Melanomen untersucht. HAF

Quelle: Veranstaltung der Roche Pharma AG und der Roche Diagnostics GmbH

\section{Effektive Testosteronsuppression mit GnRH-Antagonisten}

\begin{abstract}
Die Testosteronserumkonzentration muss bei hormonsensitivem Prostatakarzinom auf einen Wert unterhalb des Kastrationsniveaus supprimiert werden.
\end{abstract}

GnRH (Gonadotropin-Releasing-Hormon)Antagonisten wie Degarelix (Firmagon ${ }^{\circledast}$ ) sind seit circa einem Jahr eine Alternative zu den GnRH-Agonisten für die Therapie des Prostatakarzinoms. Kurt Miller, Berlin, zufolge hat die neue Substanzklasse Vorteile: Bei GnRH-Antagonisten sind im Gegensatz zu den GnRH-Agonisten keine Flareup-Phänomene bzw. Microsurges - kurzzeitige Anstiege des Testosteronserumwerts - zu beobachten. Auch wird bei 13\% bis $46 \%$ der Patienten mittels der älteren Substanzen nicht das derzeit angestrebte Kastrationsniveau von <20ng/dl Serumtestosteron erreicht.

Einer aktuellen Auswertung der zulassungsrelevanten Phase-III-Studie mit Degarelix zufolge wurde unter Degarelix im Studienzeitraum von 12 Monaten ein deutlich längeres PSA (prostataspezifisches Antigen)-progressionsfreies Überleben im Vergleich zu Leuprorelin erreicht (Crawford ED et al., 2010, J Urol 183 [Suppl]: \#670). Dies muss aber noch in Studien mit diesem Zielparameter bestätigt werden, so Fritz $\mathrm{H}$. Schröder, Rotterdam/Niederlande. RFR

Quelle: Veranstaltung der Ferring Arzneimittel $\mathrm{GmbH}$

\section{Strahlentherapie und} Selenserumkonzentration Eine deutliche Reduktion der Selenserumkonzentration nach der Strahlentherapie ist das Ergebnis einer prospektiven Studie mit 209 Mammakarzinompatientinnen, die sich einer solchen Therapie unterziehen mussten (Franca CA et al., 2010, Ann Oncol: Epub ahead of print; Information der biosyn Arzneimittel GmbH): Die mittlere Selenserumkonzentration betrug vor der Therapie 86,4 $\mathrm{g} / \mathrm{l}$, danach 47,8 $\mu \mathrm{g} / \mathrm{l}$. Die Patientinnen waren überwiegend Nichtraucher und tranken nicht, etwa die Hälfte der Patientinnen hatte zuvor noch keine Chemotherapie erhalten. Das Ergebnis legt den Schluss nahe, den Ernährungszustand der Patienten speziell mit diesem Mikronährstoff und anderen antioxidativen Stoffen im Auge zu behalten. 